\title{
In vitro efficacy of glutaraldehyde-crosslinked chitosan microspheres against the fish-pathogenic ciliate Philasterides dicentrarchi
}

\author{
A. Paramá ${ }^{1}$, A. Luzardo ${ }^{2}$, J. Blanco-Méndez ${ }^{2}$, M. L. Sanmartín ${ }^{1}$, J. Leiro ${ }^{1, *}$ \\ ${ }^{1}$ Laboratorio de Parasitología, Instituto de Investigación y Análisis Alimentarios, and ${ }^{2}$ Departamento de Farmacia \\ y Tecnología Farmacéutica, Facultad de Farmacia, Universidad de Santiago de Compostela, \\ 15782 Santiago de Compostela, Spain
}

\begin{abstract}
Philasterides dicentrarchi is a protozoan ciliate which causes significant economic losses in fish aquaculture. This study investigated the effects of chitosan microspheres cross linked with glutaraldehyde and containing beta-cyclodextrin $(\beta C D)$ on the survival of this parasite in $7 \mathrm{~d}$ cultures. When used alone in assays, neither chitosan nor $\beta C D$ showed any activity, whereas free glutaraldehyde was strongly toxic to the parasite. Microspheres were likewise strongly toxic, at total glutaraldehyde concentrations much lower than with free glutaraldehyde: near- $100 \%$ ciliate death was obtained (1) with $50 \mu \mathrm{g} \mathrm{ml}^{-1}$ of microspheres prepared with $5 \%$ glutaraldehyde and no $\beta C D$, or (2) with $10 \mu \mathrm{g} \mathrm{ml}^{-1}$ of microspheres prepared with $0.15 \%$ glutaraldehyde and $0.1 \%$ BCD. This suggests that the main active component is glutaraldehyde, but that the presence of small amounts of $\beta C D$ enhances efficacy. This high efficacy, together with the low toxicity to fish and rapid biodegradability of the individual components, suggest that these microspheres may be an attractive alternative to the formaldehyde baths traditionally used for the control of this parasite.
\end{abstract}

KEY WORDS: Ciliates - Microparticle - Chitosan - Cyclodextrins · Scuticociliatosis control · Glutaraldehyde Resale or republication not permitted without written consent of the publisher

\section{INTRODUCTION}

Ciliates are free-living in water and soil or live on the surface of or inside animal hosts in a variety of symbiotic relations (Lom \& Dyková 1992). The histiophagous scuticociliate Philasterides dicentrarchi, is a facultative parasite that causes fatal scuticociliatosis in farmed fishes such as the turbot Scophthalmus maximus (Iglesias et al. 2001) or the sea bass Dicentrarchus labrax (Dragesco et al. 1995). In view of the high virulence and endoparasitic nature of $P$. dicentrarchi, trophozoites of this ciliate cannot be effectively controlled by formalin baths while on the host, and no systemic chemotherapeutic treatments have yet proven effective (Iglesias et al. 2002); however, $P$. dicentrarchi can be readily eliminated by formalin baths while it remains in the external environment
(Paramá et al. 2003). Formalin is commonly used to treat ectoparasitic infections (Fajer-Avila et al. 2003), particularly protozoa, since it has powerful microbicidal and pesticidal effects, and binds to protein or dissolves in parasite lipids. However, it is carcinogenic and highly toxic to animals (Starr 1990).

Chitosan is a cationic polysaccharide obtained from deacetylation of chitin, a structural polymer abundant in crustaceans. Due to its biocompatibility, biodegradability, and low toxicity, chitosan is an attractive biopolymer for a variety of pharmaceutical applications (Paul \& Sharma 2000).

Cyclodextrins (CDs) are pharmacological excipients frequently used with the aim of increasing the solubility and bioavailability of poorly hydrosoluble drugs, and for protecting such drugs against oxidation, hydrolysis and photodecomposition (Blanco-Puente et 
al. 2002). For example, CDs have been used to enhance the oral absorption and efficacy of drugs against protozoans such as Leishmania (Demicheli et al. 2004). Recently, CDs have been shown to have antiprotozoal effects in experimental cryptosporidial infections (Castro-Hermida et al. 2000, 2004a,b), and have been used in the prevention and treatment of natural Cryptosporidium parvum infections in lambs and calves (Castro-Hermida et al. 2001a,b, 2004b).

The initial aim of the present study was to evaluate the possible efficacy of cyclodextrins against Philasterides dicentrarchi in in vitro culture. Free CDs had no effect, so we next tested CDs contained within glutaraldehyde-crosslinked chitosan microspheres. These showed high efficacy, and our results suggested (a) that this is basically due to the glutaraldehyde, not to the CDs (2) that the microsphere formulation is more effective than free glutaraldehyde, and (3) that the inclusion of small amounts of CD in the formulation for some reason enhances efficacy.

\section{MATERIALS AND METHODS}

Ciliate culture. Axenic culture of the ciliate Philasterides dicentrarchi was performed as previously described (Iglesias et al. 2003). Briefly, ciliates were isolated from ascitic fluid in the body cavity of turbot with scuticociliatosis from a fish farm in Galicia (NW Spain), and cultivated axenically at $18^{\circ} \mathrm{C}$ in complete L-15 medium (Sigma) containing $90 \mathrm{mg} \mathrm{l}^{-1}$ each of adenosine, cytidine and uridine, $150 \mathrm{mg} \mathrm{l}^{-1}$ guanosine, $5 \mathrm{~g} \mathrm{l}^{-1}$ glucose, $400 \mathrm{mg} \mathrm{l}^{-1} \mathrm{~L}-\alpha$-phosphatidylcholine, $200 \mathrm{mg} \mathrm{l}^{-1}$ Tween 80, $10 \%$ heat-inactivated foetal bovine serum (FBS; Sigma), 100 units $\mathrm{ml}^{-1}$ penicillin $\mathrm{G}$, $0.1 \mathrm{mg} \mathrm{ml}^{-1}$ streptomycin sulphate, and $0.25 \mathrm{mg} \mathrm{ml}^{-1}$ amphotericin B (all from Sigma). The ciliates were subcultured weekly. Prior to assays, the ciliates were washed 3 times by centrifugation $(650 \times g$ for $5 \mathrm{~min})$ and resuspended in incomplete L-15 medium (i.e. L-15 medium without nucleosides, glucose, lipids or FBS).

Preparation of microspheres. Microspheres were prepared as previously described (Ganza-González et al. 1999). Briefly, aqueous dispersions of polymer $(1 \%$ w/w; Chitosan ${ }^{\circledR} \mathrm{Cl} 210$, Pronova Biomedical) containing different concentrations of the crosslinking agent glutaraldehyde (Sigma Chemical) and beta-cyclodextrin $\left(\beta C D ;\right.$ Klentose ${ }^{\circledR}$, Roquette) (see Table 1) were maintained by shaking for $10 \mathrm{~min}$. They were then spray-dried (Buchi 190 mini spray dryer) at a flow rate of $2 \mathrm{ml} \mathrm{min}{ }^{-1}$, inlet air temperature of $110^{\circ} \mathrm{C}$, and outlet air temperature of $70^{\circ} \mathrm{C}$. The microspheres obtained were maintained at room temperature until use. For anti-ciliate activity assays, the microspheres were suspended in dimethylsulfoxide (DMSO; Sigma) immedi- ately before the assay, and the resultant stock was diluted in complete L-15 medium to obtain the required assay concentration (10 or $50 \mu \mathrm{g} \mathrm{ml}^{-1}$ ).

Determination of anti-ciliate activity. The anti-ciliate activity of the microspheres was assayed basically as described previously (Iglesias et al. 2002), with minor modifications. Ciliates in the exponential phase of culture were concentrated by centrifugation at $650 \times$ $g$ for $5 \mathrm{~min}$ and then resuspended in incomplete L-15 medium. After counting in a haemocytometer, 500 ciliates in $1 \mathrm{ml}$ of complete L-15 medium were added to each well of 24-well polystyrene plates containing 10 or $50 \mathrm{mg} \mathrm{ml}^{-1}$ of free CD or chitosan-CD microspheres. Wells containing ciliates in complete L-15 medium without $\mathrm{CD}$ or chitosan were also assayed as negative controls. To rule out possible effects of the DMSO in preparations with DMSO as solvent, assays were performed in wells with complete L-15 medium containing corresponding concentrations of DMSO ( 0.1 or $0.5 \%$, as in the 10 and $50 \mu \mathrm{g} \mathrm{ml}^{-1}$ respectively). Plates were incubated at $18^{\circ} \mathrm{C}$ for $7 \mathrm{~d}$ and ciliates were then counted in a haemacytometer. To investigate possible anti-ciliate activity of the cross-linker glutaraldehyde alone, assays were performed in wells containing ciliates incubated for $24 \mathrm{~h}$ at $18^{\circ} \mathrm{C}$ with glutaraldehyde at concentrations between $2.65 \times 10^{-5}$ and $2.65 \times 10^{-4} \mathrm{~g}$ $\mathrm{ml}^{-1}$. After incubation, viability was determined on the basis of ciliate motility, using an inverted microscope with phase-contrast illumination (Iglesias et al. 2002).

Experimental design and statistical analysis. Having demonstrated the efficacy of glutaraldehydecrosslinked chitosan- $\beta C D$ microspheres (see 'Results'), a $2 \times 2$ factorial design was used (Paniagua et al. 1998, Box et al. 1999) to identify optimal glutaraldehyde and $\beta C D$ proportions; chitosan microspheres were made up with a glutaraldehyde concentration of $0.15,5.15$ or $10.15 \%$, and a $\beta C D$ concentration of $0.1,0.2$ or $0.3 \%$ (Table 1). Effects on ciliate survival were analysed by analysis of variance, performed with the aid of Statgraphics Plus for Windows version 1.2 (Statistical Graphics Corporation).

\section{RESULTS}

\section{In vitro activity of cyclodextrins and chitosan- $\beta C D$ microspheres against Philasterides dicentrarchi}

We first investigated the possible effects of free cyclodextrins (alpha, beta or gamma cyclodextrin; 10 or $50 \mu \mathrm{g} \mathrm{ml}^{-1}$ ) on Philasterides dicentrarchi survival. As shown in Fig. 1a, none of the cyclodextrins showed significant anti-parasite effect. However, $P$. dicentrarchi survival was lowest in cultures treated with $\beta C D$, so we selected this cyclodextrin for subsequent experiments. 
Table 1. Summary of the coding system used in the factorial experimental design to investigate the effects of glutaraldehyde (GLU) and beta-cyclodextrin ( $\beta C D$ ) concentrations on anti-parasite efficacy (see also text and Fig. 2)

\begin{tabular}{|lcc|}
\hline Coded value & GLU $(\%)$ & $\beta C D(\%)$ \\
\hline-1 & 0.15 & 0.1 \\
0 & 5.15 & 0.2 \\
1 & 10.15 & 0.3 \\
\multicolumn{2}{c}{ Doding } & $V_{\mathrm{n}}=V_{0}+\left(\Delta V_{\mathrm{n}} V_{\mathrm{c}}\right)$ \\
$V_{\mathrm{c}}=\left(V_{\mathrm{n}}-V_{0} / \Delta V_{\mathrm{n}}\right.$ & \\
$V_{\mathrm{c}}=$ coded value & \\
$V_{\mathrm{n}}=$ natural value & \\
$V_{0}=$ natural value in the centre of the domain \\
$\Delta V_{\mathrm{n}}=$ increment in $V_{\mathrm{n}}$ corresponding to 1 unit of $V_{\mathrm{c}}$ \\
\hline
\end{tabular}
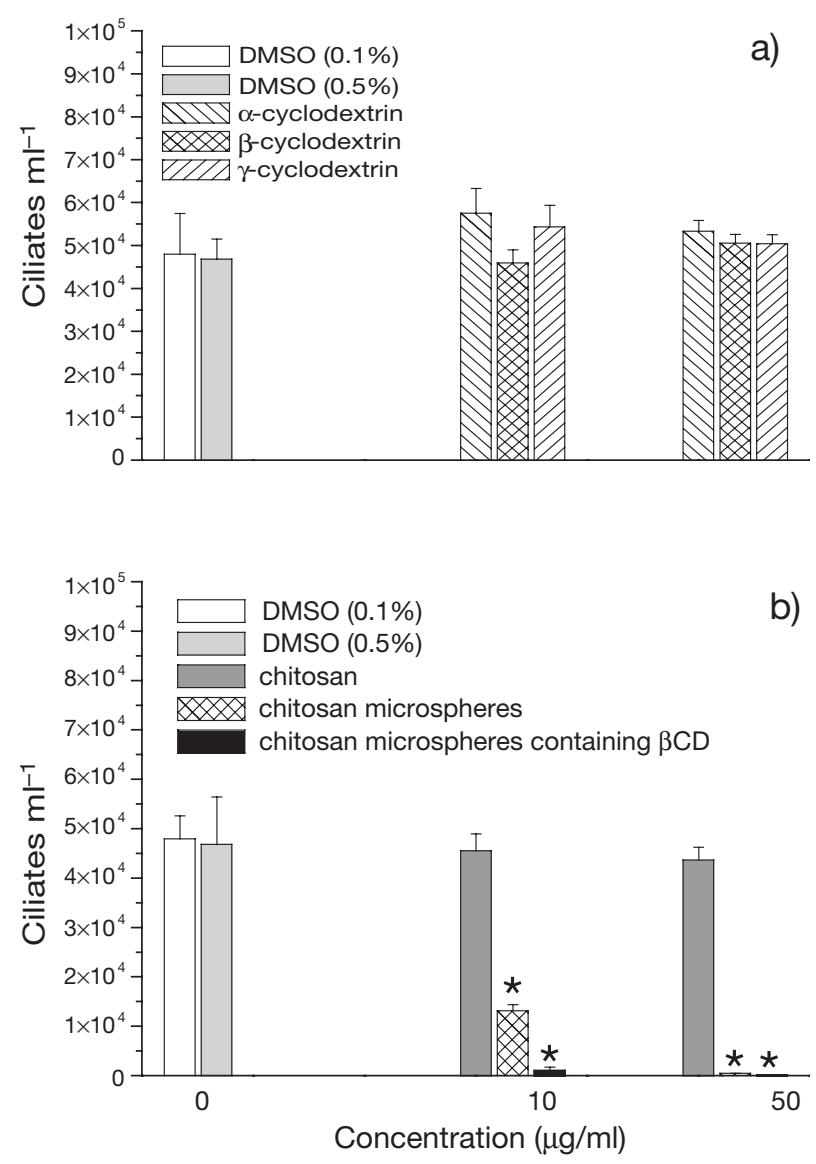

Fig. 1. Effects of (a) free cyclodextrins and (b) free chitosan, glutaraldehyde-linked chitosan microspheres with or without beta-cyclodextrin ( $\beta C D$ ) on Philasterides dicentrarchi survival in culture. Microspheres were prepared with $5 \%$ glutaraldehyde and 0 or $0.2 \% \beta C D$, and were added to the $7 \mathrm{~d}$ cultures at 10 or $50 \mu \mathrm{g} \mathrm{ml}^{-1}$. Bars $=$ means $\pm \operatorname{SEM}(\mathrm{n}=5) .{ }^{*} \mathrm{p}<0.01$ with respect to controls incubated in the presence of dimethylsulfoxide (DMSO) at $0.1 \%$ (microspheres at $10 \mu \mathrm{g} \mathrm{ml}^{-1}$ ) or DMSO at $0.5 \%$ (microspheres at $10 \mu \mathrm{g} \mathrm{ml}^{-1}$ )
We next evaluated the effects of chitosan microspheres containing $\beta C D$ on Philasterides dicentrarchi survival. Microspheres were initially prepared with $0.2 \% \beta C D$ and $5 \%$ glutaraldehyde as crosslinking agent. Both microspheres without $\beta C D$ and microspheres containing $\beta C D$ led to a marked and significant decline in $P$. dicentrarchi survival, at both microsphere concentrations tested (10 and $50 \mu \mathrm{g} \mathrm{ml}^{-1}$ ); the decline obtained with $\beta C D$-containing microspheres was significantly greater (Fig. 1b). Chitosan alone without glutaraldehyde had no significant effects on $P$. dicentrarchi survival (Fig. 1b).

\section{Optimal glutaraldehyde and $\beta C D$ proportions in chitosan- $\beta C D$ microspheres}

Observed and modelled effects of glutaraldehyde (GLU) and $\beta C D$ proportions on ciliate survival are shown in Tables $2 \& 3$, for microspheres at 10 and $50 \mu \mathrm{g}$ $\mathrm{ml}^{-1}$ respectively. On the basis of estimation of statistical significance of model coefficients ( $\alpha=0.2$, Student's $t$-tests), the effects of these 2 variables on ciliate survival can be described by the following equations:

$$
\begin{aligned}
& \text { No. ciliates } \mathrm{ml}^{-1}=83333.33-7100 \mathrm{GLU} \times \beta \mathrm{CD} \\
& {\left[\text { for microspheres at } 10 \mu \mathrm{g} \mathrm{ml}^{-1}\right. \text { ] }} \\
& \text { No. ciliates } \mathrm{ml}^{-1}=1633.33-1700 \mathrm{GLU} \times \beta \mathrm{CD} \\
& {\left[\text { for microspheres at } 50 \mu \mathrm{g} \mathrm{ml}^{-1}\right. \text { ] }}
\end{aligned}
$$

where GLU and $\beta C D$ are the proportions (\%) of glutaraldehyde and $\beta-C D$ respectively.

In the first model (microspheres at $10 \mu \mathrm{g} \mathrm{ml}^{-1}$ ), the individual effects of GLU and $\beta C D$ were not significant at the $80 \%$ level, but the cross-term GLU $\times \beta C D$ was significant (Table 2). In the second model (microspheres at $50 \mu \mathrm{g} \mathrm{ml}^{-1}$ ), both individual effects and the cross-term were significant at the $80 \%$ level (or very nearly so; $\beta C D, p=0.2056$ ) (Table 3 ).

Response-surface plots of the 2 models are shown in Fig. 2. These plots are rather difficult to interpret, suggesting that optimal activity is obtained with either low-glutaraldehyde/low- $\beta C D$ or high-glutaraldehyde/ high-ßCD.

Fig. 3 summarizes the effects of chitosan microspheres without $\beta C D$ on Philasterides dicentrarchi survival. As can be seen, increasing glutaraldehyde concentration led to a dose-dependent increase in efficacy against $P$. dicentrarchi. The highest efficacy was obtained with $50 \mu \mathrm{g} \mathrm{ml} \mathrm{m}^{-1}$ of microspheres containing $5.15 \%$ glutaraldehyde.

Fig. 4 summarizes the effects of saltwater pretreatment of chitosan microspheres $\left(50 \mu \mathrm{g} \mathrm{ml}^{-1} ; 5.15 \%\right.$ glutaraldehyde) or chitosan- $\beta C D$ microspheres $\left(10 \mu \mathrm{g} \mathrm{ml}^{-1} ; 0.15 \%\right.$ glutaraldehyde, $0.1 \% \beta C D$ ) on Philasterides dicentrarchi survival. Microspheres were assayed against $P$. dicen- 
Table 2. Regression analysis of the data obtained in the factorial experimental design to investigate the effects of glutaraldehyde $(\mathrm{GLU})$ and beta-cyclodextrin $(\beta C D)$ concentrations on anti-parasite efficacy (see also text, Table 1 and Fig. 2). Data for microspheres at $10 \mu \mathrm{g} \mathrm{ml}^{-1}$

\begin{tabular}{|c|c|c|c|c|c|c|c|c|}
\hline GLU & $\beta C D$ & $\begin{array}{l}\text { Observed } \\
\text { value }\end{array}$ & $\begin{array}{l}\text { Fitted } \\
\text { value }\end{array}$ & Term & Coefficient & $\begin{array}{l}\text { Sum of } \\
\text { squares }\end{array}$ & $F$-ratio & $\mathrm{p}$-value \\
\hline 0 & 0 & 3000 & 8333.33 & Constant & 8333.33 & & & \\
\hline 1 & 1 & 6000 & 3783.33 & GLU & -600 & $1.44 \times 10^{6}$ & 0.05 & 0.8476 \\
\hline-1 & -1 & 900 & -1316.67 & $\beta C D$ & 3150 & $3.97 \times 10^{7}$ & 1.31 & 0.3709 \\
\hline 1 & -1 & 13900 & 11683.3 & $\mathrm{GLU} \times \mathrm{bCD}$ & -7100 & $2.02 \times 10^{8}$ & 6.66 & 0.1231 \\
\hline 0 & 0 & 4800 & 8333.33 & Total error & & $6.06 \times 10^{7}$ & & \\
\hline-1 & 1 & 21400 & 19183.3 & Total (corr.) & & $3.03 \times 10^{8}$ & & \\
\hline \multicolumn{9}{|c|}{$\begin{array}{l}\mathrm{R}^{2}=80.0288 \% \\
\mathrm{R}^{2} \text { (adjusted) }=50.072 \% \\
\mathrm{SE}=5503.79\end{array}$} \\
\hline
\end{tabular}

Table 3. Results of regression analysis of the data obtained in the factorial experimental design to investigate the effects of glutaraldehyde (GLU) and $\beta C D$ concentrations on anti-parasite efficacy (see also text, Table $1 \&$ Fig. 2). Data for microspheres at $50 \mu \mathrm{g} \mathrm{ml}$

\begin{tabular}{|c|c|c|c|c|c|c|c|c|}
\hline GLU & $\beta C D$ & $\begin{array}{l}\text { Observed } \\
\text { value }\end{array}$ & $\begin{array}{l}\text { Fitted } \\
\text { value }\end{array}$ & Term & Coefficient & $\begin{array}{l}\text { Sum of } \\
\text { squares }\end{array}$ & $F$-ratio & $\mathrm{p}$-value \\
\hline 0 & 0 & 300 & 1633.33 & Constant & 1633.33 & & & \\
\hline 1 & 1 & 400 & -216.67 & GLU & -1700 & $1.16 \times 10^{7}$ & 5.04 & 0.1538 \\
\hline-1 & -1 & 1000 & 383.33 & $\beta C D$ & 1400 & $7.84 \times 10^{6}$ & 3.42 & 0.2056 \\
\hline 1 & -1 & 700 & 83.33 & $G L U \times \beta C D$ & -1500 & $9.61 \times 10^{6}$ & 4.19 & 0.1772 \\
\hline 0 & 0 & 500 & 1633.33 & Total error & & $4.58 \times 10^{6}$ & & \\
\hline-1 & 1 & 6900 & 6283.33 & Total (corr.) & & $3.36 \times 10^{7}$ & & \\
\hline \multicolumn{9}{|c|}{$\begin{array}{l}\mathrm{R}^{2}=80.0288 \% \\
\mathrm{R}^{2} \text { (adjusted) }=50.072 \% \\
\mathrm{SE}=5503.79 \\
\text { Durbin Watson statistic }=2.03237\end{array}$} \\
\hline
\end{tabular}
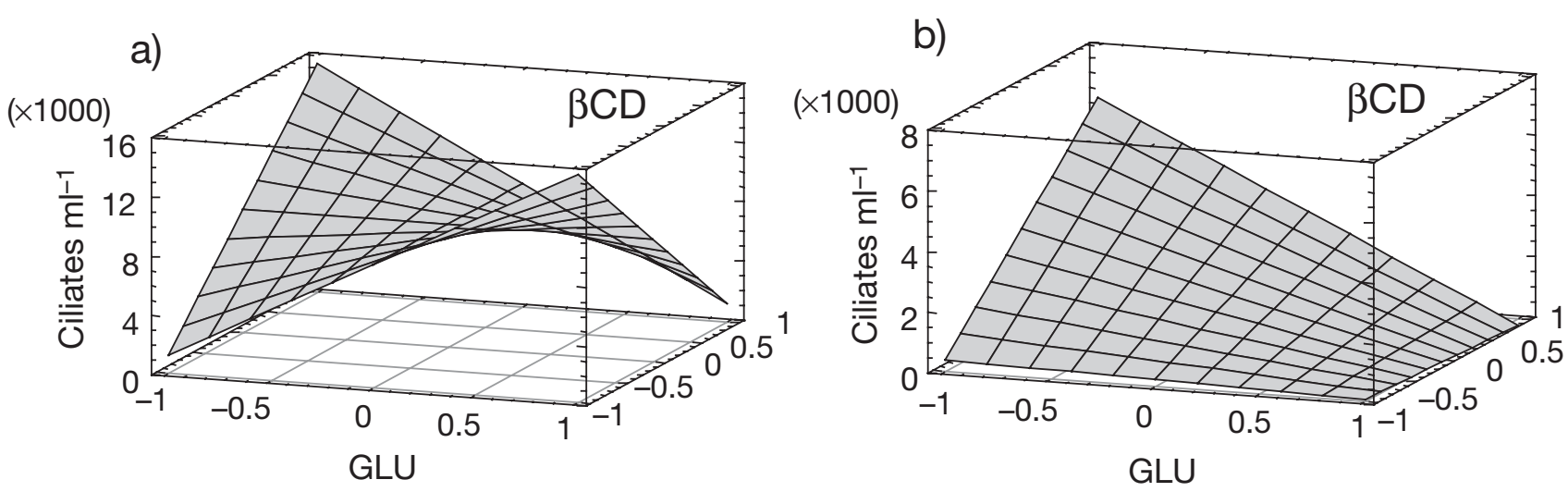

Fig. 2. Response-surface plots derived by regression analysis of the data obtained in the factorial-design assays to investigate the effects of glutaraldehyde (GLU) and beta-cyclodextrin ( $\beta C D$ ) concentrations on ciliate survival. Plots are shown for microspheres at (a) $10 \mu \mathrm{g} \mathrm{ml}^{-1}$ and (b) $50 \mu \mathrm{g} \mathrm{ml}{ }^{-1}$. Glutaraldehyde concentrations were $0.15 \%$ (coded value -1 ), $5.15 \%$ (coded value 0 ) and $10.15 \%$ (coded value 1 ); $\beta C D$ concentrations were $0.1 \%$ (coded value -1 ), $0.2 \%$ (coded value 0 ) and $0.3 \%$ (coded value 1 )

trarchi either with or without prior maintenance for $1 \mathrm{wk}$ in sterile seawater. As can be seen from Fig. 4, both non-pretreated chitosan microspheres and nonpretreated chitosan- $\beta C D$ microspheres showed strong anti-parasite activity. Seawater pretreatment markedly reduced these activities, but the anti-parasite activity remaining was still considerable (ciliate survival of about $20 \%$ in untreated control cultures). 


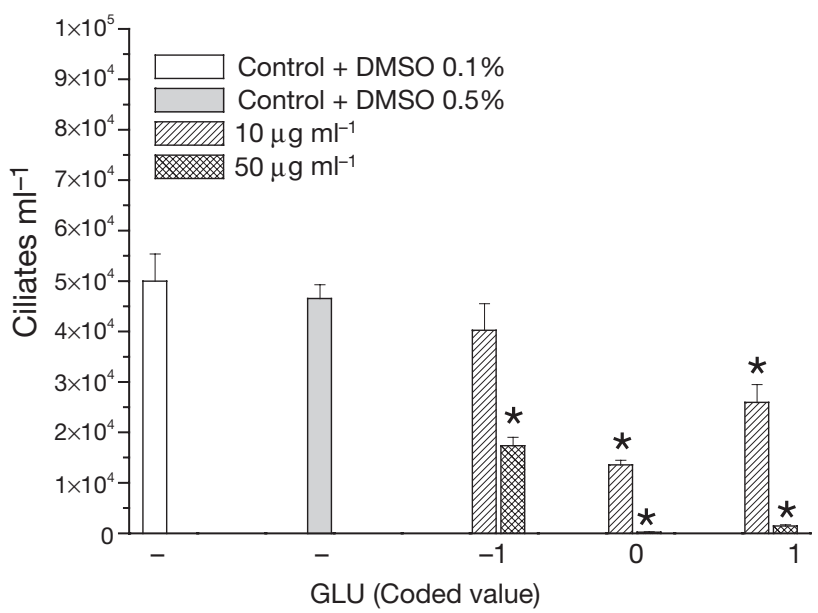

Fig. 3. Effects of glutaraldehyde-linked chitosan microspheres without beta-cyclodextrin $(\beta C D)$ on Philasterides dicentrarchi survival in culture. Glutaraldehyde (GLU) concentrations were $0.15 \%$ (coded value -1 ), $5.15 \%$ (coded value 0 ) and $10.15 \%$ (coded value 1 ). Microsphere concentration was 10 or $50 \mu \mathrm{g} \mathrm{ml}^{-1}$. Bars represent means \pm SEM $(\mathrm{n}=5)$. ${ }^{*} \mathrm{p}<$ 0.01 with respect to controls incubated in the presence of dimethylsulfoxide (DMSO) at $0.1 \%$ (microspheres at $10 \mu \mathrm{g}$ $\mathrm{ml}^{-1}$ ) or DMSO at $0.5 \%$ (microspheres at $50 \mu \mathrm{g} \mathrm{ml} \mathrm{m}^{-1}$ )

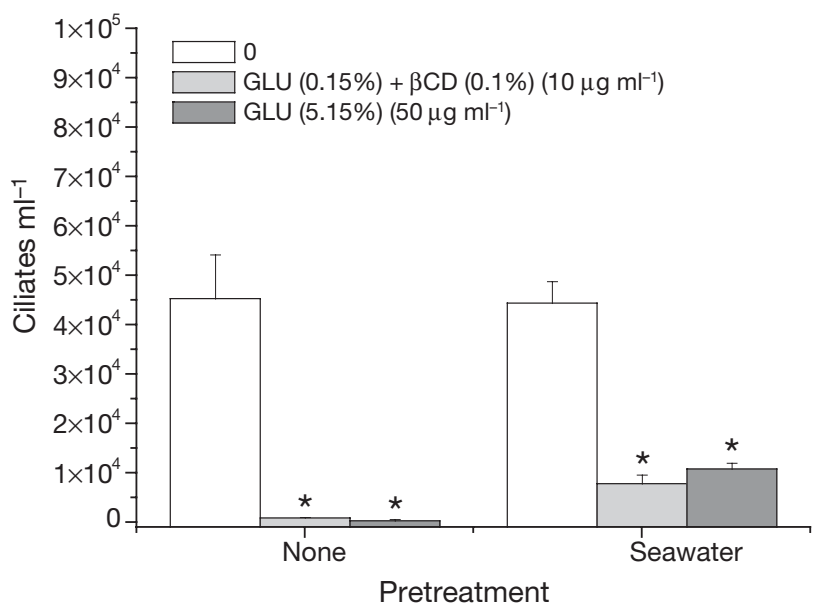

Fig. 4. Effects of $1 \mathrm{wk}$ maintenance in seawater on antiparasite efficacy of chitosan microspheres $(0.15 \%$ glutaraldehyde [GLU] and $0.1 \%$ beta-cyclodextrin ( $\beta C D)$, or $5.15 \%$ glutaraldehyde and no $\beta C D)$. Bars $=$ means \pm SEM $(n=5)$

\section{In vitro activity of glutaraldehyde against Philasterides dicentrarchi}

The effects of different glutaraldehyde concentrations on Philasterides dicentrarchi survival are shown in Fig. 5. At concentrations of $1.325 \times 10^{-4} \mathrm{~g} \mathrm{~m}^{-1}$ or more, with incubation for $24 \mathrm{~h}$, glutaraldehyde killed all ciliates in the culture.

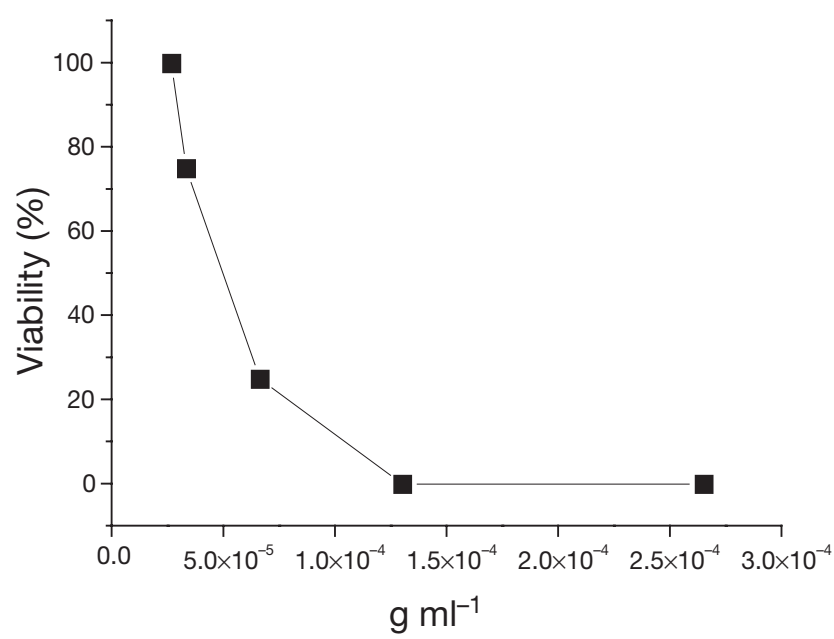

Fig. 5. Effects of free glutaraldehyde $\left(\mathrm{g} \mathrm{ml}^{-1}\right)$ on the survival of Philasterides dicentrarchi trophozoites in $24 \mathrm{~h}$ microcultures

\section{DISCUSSION}

To date, no effective treatments have been described for systemic scuticociliatosis due to Philasterides dicentrarchi in farmed turbot. However, these infections can be effectively prevented by formaldehyde baths, which rapidly kill the free-living ciliate at formaldehyde doses as low as $62 \mathrm{ppm}$ (Iglesias et al. 2002). We recently reported an in vitro screening study of the efficacy of diverse antiprotozoals against $P$. dicentrarchi (Iglesias et al. 2002), identifying several promising compounds; however, these compounds may be difficult to administer, and may show significant toxicity to fish. Furthermore, pollution problems derived from pathogen-control treatments in aquaculture environments are a cause of increasing concern (Ernst et al. 2001). Anti-ectoparasitic chemicals, including formaldehyde, have been implicated in water pollution (O'Connor \& Huggett 1987), and many candidate chemotherapeutic chemicals have not been accepted for use in aquaculture by regulatory authorities (Iglesias et al. 2002). Finally, the efficacy of many candidate chemotherapeutical chemicals is markedly reduced in seawater (Iglesias et al. 2002).

The initial aim of the present study was to evaluate possible antiprotozoal activities of various cyclodextrins $(\alpha C D, \beta C D$, and $\gamma C D)$. CDs are cyclical sugar polymers that solubilize a range of hydrophobic compounds within their hydrophobic core (Kritharides et al. 1996), and which in previous studies have been shown to have significant antiprotozoal activity (Castro-Hermida et al. 2000). We found that an addition of free CDs to Philasterides dicentrarchi culture media at $\mu \mathrm{g} \mathrm{ml}^{-1}$ concentrations (i.e. concentrations not significantly affecting medium molarity; results not 
shown) did not affect $P$. dicentrarchi survival. It is well known that CDs can interact specifically with phospholipids (Irie et al. 1992) and precipitate lipoproteins (Sharma \& Janis 1991), and the culture medium used in our assays (L-15) contains L- $\alpha$-phosphatidylcholine (Iglesias et al. 2003), which may have interacted with and inactivated the free CDs. Lipoproteins contained in the foetal bovine serum included in the medium may have had a similar effect.

In view of these results, we decided to evaluate the efficacy of $\beta C D$ contained within chitosan microspheres. Independently of possible toxic effects on the ciliate, microspheres have previously been found to stimulate phagocytosis by phagotropic fish parasites (Lom \& Dyková 1992). These assays indicated that $\beta C D$-containing chitosan microspheres indeed showed significant anti-parasite activity.

The production of chitosan microspheres requires the use of a cross-linking agent (here glutaraldehyde), the concentration of which affects the permeability and hardness of the microsphere and, thus, both its digestibility by phagocytic organisms and its drugrelease properties. To investigate the anti-parasite activity of microspheres prepared with different concentrations of $\beta C D$ and glutaraldehyde, we performed an additional series of assays using a factorial design, as used in previous studies to optimize the formulation of chitosan microspheres (Ko et al. 2003). The results of these assays showed that when microspheres were added to the medium at a low concentration $(10 \mu \mathrm{g}$ $\mathrm{ml}^{-1}$ ), anti-parasite activity was not significantly affected at the $80 \%$ level by either factor independently ( $\beta C D$ concentration or glutaraldehyde concentration), but was significantly affected by the crossterm of the 2 factors. When microspheres were added to the medium at the higher concentration $\left(50 \mu \mathrm{g} \mathrm{ml}^{-1}\right)$, anti-parasite activity was significantly affected at the $80 \%$ level by both factors individually, and by the cross-term. At both microsphere concentrations, good anti-parasite activity was obtained with low glutaraldehyde concentration $(0.15 \%)$ and low $\beta C D$ concentration $(0.1 \%)$.

In view of the results, additional trials were performed with glutaraldehyde-crosslinked microspheres without $\beta C D$. The results of these trials are not easy to reconcile with the results summarized in Fig. 2, in that increasing glutaraldehyde concentration led to increasing anti-parasite activity (cf. Fig. 2, in which increasing glutaraldehyde concentration while maintaining $\beta C D$ constant at its lowest concentration led to declining anti-parasite activity). What is clear from these results is that glutaraldehyde-linked chitosan microspheres show strong anti-parasite activity, indicating that the main active component is glutaraldehyde; but at the same time, the efficacy of glutaral- dehyde-crosslinked microspheres appears to be enhanced in some way by inclusion of small amounts of $\beta C D$ in the formulation.

These results suggest that the observed antiparasite activity is fundamentally due to toxicity of glutaraldehyde released after ingestion of microspheres by the parasite. The possible decline in activity at higher glutaraldehyde concentrations (Fig. 3; but see also Fig. 2) may reflect increased hardness of the microspheres, reducing digestibility in the ciliate's food vacuole. Glutaraldehyde added directly to the culture medium caused death of all ciliates at concentrations of $1.35 \times 10^{-4} \mathrm{~g} \mathrm{ml}^{-1}$ or higher. Bearing in mind that chitosan microspheres are about $3 \mu \mathrm{m}$ in diameter, and that optimal anti-parasite activity (in microspheres without $\beta C D$ ) was obtained with a glutaraldehyde concentration of $5 \%$, the concentration of glutaraldehyde in medium containing microspheres at $50 \mu \mathrm{g} \mathrm{ml}^{-1}$ (causing death of $99 \%$ of ciliates) can be estimated as about $35 \times 10^{-9} \mathrm{\mu g} \mathrm{ml}^{-1}$; thus the total glutaraldehyde concentration required for near- $100 \%$ ciliate death is about $3 \times 10^{5}$ times lower for microspheres than for free glutaraldehyde. Near-100\% ciliate death was also obtained with $10 \mu \mathrm{g} \mathrm{ml}^{-1}$ of microspheres prepared with $0.15 \%$ glutaraldehyde and $0.1 \% \beta C D$; i.e. total glutaraldehyde concentration about 170 times lower again, in other words about $5 \times$ $10^{7}$ times lower than for free glutaraldehyde. Again, these findings support the view that the principal active component of these formulations is glutaraldehyde, but that the presence of small amounts of $\beta C D$ in some way enhances efficacy.

Activity trials performed with microspheres that had been stored in seawater for $1 \mathrm{wk}$ indicated that seawater significantly reduced anti-parasite activity, but that strong activity nevertheless was remained.

Glutaraldehyde is toxic to saltwater fish; however, it is markedly less toxic than formaldehyde (the standard bath treatment for scuticociliatosis), and is rapidly biodegraded in marine environments (Leung 2001). Furthermore, bath treatment with free formaldehyde evidently requires a much higher total concentration of aldehyde than bath treatment with glutaraldehydecrosslinked microspheres. Chitosan shows low or negligible toxicity and rapid biodegradability (Tharanathan \& Kittur 2003), as do CDs (Brusseau et al. 1994); indeed, CDs have been used in environmental remediation in view of their capacity to solubilize and desorb contaminants (Boving et al. 1999, Luo et al. 2003). In addition, microsphere preparations can be readily removed from tanks by decantation after the required exposure period. These considerations all suggest that glutaraldehyde-crosslinked chitosan microspheres will show relatively low toxicity to fish and relatively low environmental toxicity. 
In conclusion, our results indicate that glutaraldehyde-crosslinked chitosan microspheres containing $\beta C D$ may be an attractive alternative to formaldehyde baths for the treatment of fish scuticociliatosis due to Philasterides dicentrarchi. Our results suggest that the most promising approach may be to use chitosan microspheres with low glutaraldehyde content $(\sim 0.15 \%)$ and low $\beta C D$ content $(\sim 0.1 \%)$, which seem to offer high efficacy at low microsphere concentrations. However, the precise mechanisms by which the different components contribute to the observed activity remain unclear. A priori considerations suggest that the microspheres will have relatively low toxicity to fish and low environmental toxicity, but this will of course need to be confirmed by in vivo trials. Finally, microspheres of this type are clearly much more expensive than formaldehyde, and it remains to be seen whether they will prove cost-effective. Tank trials are currently underway to test the utility and costeffectiveness of these microsphere preparations in vivo.

Acknowledgements. This work was supported by grants PGIDIT02RMA23701PR and PGIDIT02RAG20301PR from the Xunta de Galicia (Spain) and AGL2003-04644 from the Comisión Interministerial de Ciencia y Tecnología (CICYT), Spain.

\section{LITERATURE CITED}

Blanco-Puente HB, Esteban-Fernández J, Blanco-Méndez J, Otero-Espinar FJ (2002) Use of $\beta$-cyclodextrins to prevent modifications of the properties of carbopol hydrogels due to carbopol-drug interaction. Chem Pharm Bull 50:40-46

Boving TB, Wang XJ, Brusseau ML (1999) Cyclodextrinenhanced solubilization and removal of residual-phase chlorinated solvents from porous media. Environ Sci Technol 33:767-770

Box GEP, Hunter WG, Hunter JS (1999) Estadística para investigadores: introducción al diseño de experimentos, análisis de datos y construcción de modelos. Reverté, Barcelona

Brusseau ML, Wang XJ, Huang QG (1994) Enhanced transport of low-polarity organic compounds through soil by cyclodextrin. Environ Sci Technol 28:952-956

Castro-Hermida JA, Freire-Santos F, Oteiza-López AM, AresMazas E (2000) Unexpected activity of beta-cyclodextrin against experimental infection by Cryptosporidium parvum. J Parasitol 86:1118-1120

Castro-Hermida JA, Quílez-Cinca J, López-Bernad F, Sánchez-Acedo C, Freire-Santos F, Ares-Mazas E (2001a) Treatment with beta-cyclodextrin of natural Cryptosporidium parvum infections in lambs under field conditions. Int J Parasitol 31:1134-1137

Castro-Hermida JA, González-Losada Y, Freire-Santos F, Mezo-Menéndez M, Ares-Mazas E (2001b) Evaluation of beta-cyclodextrin against natural infections of cryptosporidiosis in calves. Vet Parasitol 101:85-89

Castro-Hermida JA, Gomez-Couso H, Ares-Mazas ME, Gonzalez-Bedia MM, Castaneda-Cancio N, Otero-Espinar FJ, Blanco-Méndez J (2004a) Anticryptosporidial activity of furan derivative G1 and its inclusion complex with betacyclodextrin. J Pharm Sci 93:197-206

Castro-Hermida JA, Pors I, Otero-Espinar F, Luzardo-Alvarez A, Ares-Mazas E, Chartier C (2004b). Efficacy of alphacyclodextrin against experimental cryptosporidiosis in neonatal goats. Vet Parasitol 120:35-41

Demicheli C, Ochoa R, da Silva JB, Falcao CA, RossiBergmann B, de Melo AL, Sinisterra RD, Frezard F (2004) Oral delivery of meglumine antimoniate-beta-cyclodextrin complex for treatment of leishmaniasis. Antimicrob Agents Chemother 48:100-103

Dragesco A, Dragesco J, Coste F, Gasc C, Romestand B, Raymond J, Bouix G (1995) Philasterides dicentrarchi, n. sp. (Ciliophora, Scuticociliatida), a histiophagous opportunistic parasite of Dicentrarchus labrax (Linnaeus, 1758), a reared marine fish. Eur J Protistol 31:327-340

Ernst W, Jackman P, Doe K, Page F, Julien G, Mackay K, Sutherland T (2001) Dispersion and toxicity to non-target aquatic organisms of pesticides used to treat sea lice on salmon in net pen enclosures. Mar Pollut Bull 42:433-444.

Fajer-Avila EJ, Abdo-de la Parra I, Aguilar-Zarate G, Contreras-Arce R, Zaldivar-Ramirez J, Betancourt-Lozano M (2003) Toxicity of formalin to bullseye puffer fish (Sphoeroides annulatus Jenyns, 1843) and its effectiveness to control ectoparasites. Aquaculture 223:41-50

Ganza-González A, Anguiano-Igea S, Otero-Espinar FJ, Blanco-Méndez J (1999) Chitosan and chondroitin microspheres for oral-administration controlled release of metoclopramide. Eur J Pharm Biopharm 48:149-155

Iglesias R, Paramá A, Álvarez MF, Leiro J, Fernández J, Sanmartin ML (2001) Philasterides dicentrarchi (Ciliophora, Scuticociliatida) as the causative agent of scuticociliatosis in farmed turbot Scophthalmus maximus in Galicia (NW Spain). Dis Aquat Org 46:47-55

Iglesias R, Paramá A, Alvarez MF, Leiro J, Sanmartín ML (2002) Antiprotozoals effective in vitro against the scuticociliate fish pathogen Philasterides dicentrarchi. Dis Aquat Org 49:191-197

Iglesias R, Paramá A, Alvarez MF, Leiro J, Aja C, Sanmartín ML (2003) In vitro growth requirements for the fish pathogen Philasterides dicentrarchi (Ciliophora, Scuticociliatida). Vet Parasitol 111:19-30

Irie T, Fukunaga K, Pitha J (1992) Hydroxypropylcyclodextrins in parenteral use. I. Lipid dissolution and effects on lipid transfers in vitro. J Pharm Sci 81:521-523

Ko JA, Park HJ, Park YS, Hwang SJ, Park JB (2003) Chitosan microparticle preparation for controlled drug release by response surface methodology. J Microencapsul 20: 791-797

Kritharides L, Kus M, Brown AJ, Jessup W, Dean RT (1996) Hydroxypropyl-beta-cyclodextrin-mediated efflux of 7 ketocholesterol from macrophage foam cells. J Biol Chem 271:27450-27455

Leung HW (2001) Ecotoxicology of glutaraldehyde: review of environmental fate and effects studies. Ecotoxicol Environ Saf 49:26-39

Lom J, Dyková I (1992) Protozoan parasites of fishes. In: Developments in aquaculture and fisheries science, Vol 26. Elsevier, Amsterdam, p 237-288

Luo YC, Zeng QR, Wu G, Luan ZK, Yang RB, Liao BH (2003) Effect of beta-cyclodextrin compounds on the solubilization of three selected pesticides and their toxicity with methyl parathion to Rana tigrina tadpoles. Bull Environ Contam Toxicol 70:998-1005

O'Connor JM, Huggett RJ (1987) Aquatic pollution problems: North Atlantic coast, including Chesapeake Bay. Aquat Toxicol 318:28-53 
Paniagua E, Fernández J, Ortega M, Paramá A, Sanmartín ML (1998) Effects of temperature, salinity and incubation time on in vitro survival of an amoeba infecting the gills of turbot, Scophthalmus maximus. J Fish Dis 21:77-80

Paramá A, Iglesias R, Álvarez MF, Leiro J, Aja C, Sanmartín ML (2003) Philasterides dicentrarchi (Ciliophora, Scuticociliatida): experimental infection and possible routes of entry in farmed turbot (Scophthalmus maximus). Aquaculture 217:73-80

Editorial responsibility: Wolfgang Körting,

Hannover, Germany
Paul W, Sharma CP (2000) Chitosan, a drug carrier for the 21st century: a review. STP Pharm Sci 10:5-22

Sharma A, Janis LS (1991) Lipoprotein-cyclodextrin interaction. Clin Chim Acta 199:129-137

Starr TB (1990) Quantitative cancer risk estimation for formaldehyde. Risk Anal 10:85-91

Tharanathan RN, Kittur FS (2003) Chitin - the undisputed biomolecule of great potential. Crit Rev Food Sci Nutr 43: $61-87$

Submitted: April 26, 2004; Accepted: September 27, 2004 Proofs received from author(s): March 14, 2005 